La Revue

des Droits

de l'Homme

\section{La Revue des droits de l'homme}

Revue du Centre de recherches et d'études sur les droits fondamentaux

$3 \mid 2013$

Revue des droits de l'homme $-\mathrm{N}^{\circ} 3$

Dépénalisation du séjour irrégulier des étrangers : l'opiniâtre résistance des autorités françaises

(De l'arrêt El Dridi à la loi du 31 décembre 2012)

\title{
Patrick Henriot
}

\section{OpenEdition}

Journals

Édition électronique

URL : http://journals.openedition.org/revdh/426

DOI : $10.4000 /$ revdh.426

ISSN : 2264-119X

Éditeur

Centre de recherches et d'études sur les droits fondamentaux

Référence électronique

Patrick Henriot, « Dépénalisation du séjour irrégulier des étrangers : l'opiniâtre résistance des autorités françaises », La Revue des droits de l'homme [En ligne], 3 | 2013, mis en ligne le 02 janvier 2014,

consulté le 10 décembre 2020. URL : http://journals.openedition.org/revdh/426 ; DOI : https://doi.org/ $10.4000 /$ revdh.426

Ce document a été généré automatiquement le 10 décembre 2020.

Tous droits réservés 


\section{Dépénalisation du séjour irrégulier des étrangers : l'opiniâtre résistance des autorités françaises}

(De l'arrêt El Dridi à la loi du 31 décembre 2012)

\section{Patrick Henriot}

1 C'est peu dire que la décision rendue par la Cour de justice de l'Union européenne (CJUE) le 28 avril 2011 dans l'affaire El Dridi ${ }^{1}$ a produit l'effet d'un véritable coup de tonnerre parmi les défenseurs des droits des étrangers ${ }^{2}$. Peu d'entre eux avaient imaginé que la directive 2008/115/CE du 16 décembre $2008^{3}$ - qu'ils avaient surnommée "la directive de la honte»- pourrait servir de point d'appui pour s'attaquer à la pénalisation du séjour irrégulier. Il était au contraire admis, depuis longtemps, que rien de bon ne pourrait venir de cet ensemble de dispositions régressives, clairement dédié à la mise en œuvre d'une politique d'éloignement implacable.

2 Mais s'il a d'abord constitué une heureuse surprise, cet arrêt El Dridi a également marqué le point de départ de longues tribulations administratives, judiciaires et, finalement, législatives. L'onde de choc produite par ce petit séisme jurisprudentiel a d'abord été amplifiée, quelques mois plus tard, par la « réplique » qu'a constitué l'arrêt Achughbabian du 6 décembre 20114. Elle s'est ensuite propagée pendant de nombreux mois encore, occupant tout à la fois l'attention des militants associatifs, naturellement attentifs aux conséquences de ces arrêts pour les droits des étrangers et celle des avocats, magistrats, ministres, parlementaires... directement impliqués dans la réaction en chaîne qu'ils ont provoquée.

3 L'avenir dira si l'ultime épisode - la publication (au journal officiel du $1^{\mathrm{er}}$ janvier 2013) de la loi du 31 décembre 2012 « relative [notamment] à la retenue pour vérification du droit au séjour... $»^{5}$ - mettra définitivement fin aux controverses nées de la confrontation de notre législation nationale aux exigences de la directive « retour » telle qu'interprétée par la CJUE. En attendant, le moment est peut-être venu de tenter d'analyser, avec un peu de recul, les raisons pour lesquelles la jurisprudence européenne a provoqué tant 
de perturbations et rencontré tant d'obstacles au sein de nos juridictions, au point de ne pouvoir s'imposer, finalement, que par la voie législative.

Il y a deux explications, au moins, à l'importance et à la durée des secousses produites par ces arrêts. La première tient à un phénomène fortuit : l'espèce de collision qui s'est produite entre la jurisprudence de Luxembourg et la législation française régissant la "garde à vue ", que le Parlement venait au surplus de réformer profondément. La seconde tient à l'extraordinaire capacité de résistance dont nos autorités - tant politiques, qu'administratives et même judiciaires pour certaines - ont fait preuve, pour tenter de mettre le dispositif d'éloignement des étrangers en situation irrégulière hors d'atteinte de cette jurisprudence européenne et ce, alors même qu'elle le condamnait inéluctablement.

\section{Interprétation de la directive « retour » et régime de la garde à vue : un choc inattendu}

5 La "garde à vue» (mesure de contrainte au moyen de laquelle une personne soupçonnée d'avoir commis une infraction est maintenue contre son gré à la disposition des enquêteurs) était très largement utilisée pour retenir dans les locaux de police les étrangers soupçonnés d'être sans titre de séjour, infraction réprimée par l'article L 621-1 du Code de l'entrée et du séjour des étrangers et du droit d'asile (CESEDA). Pas moins de 74000 personnes avaient ainsi été placées en garde à vue, en 2010, sur le fondement de suspicions d'infractions à la législation sur le séjour.

6 Les procédures d'éloignement des étrangers en séjour irrégulier se déroulaient donc selon un processus parfaitement rodé. Elles commençaient le plus souvent par un contrôle d'identité, suivi d'une interpellation, puis d'une garde à vue de 24 à 48 heures, «justifiée » par la poursuite d'une infraction à la législation sur le séjour. La durée de cette garde à vue avait l'énorme avantage de laisser le temps à l'administration de vérifier l'identité et la situation de l'étranger et, si sa situation de séjour irrégulier était confirmée, de préparer les décisions administratives d'éloignement et de placement en rétention. Les poursuites pénales étaient alors abandonnées dès la fin de la garde à vue et le placement dans un centre de rétention administrative pouvait immédiatement lui succéder. La garde à vue, mesure de police judiciaire, était donc utilisée instrumentalisée - au profit de la procédure administrative d'éloignement, dont elle constituait en quelque sorte le préliminaire ou l'antichambre.

7 Des directives de politique pénale précisées par des circulaires des 21 février et 4 décembre 2006 avaient d'ailleurs formalisé et officialisé ces orientations pratiques en prescrivant aux procureurs de la République «de n'exercer l'action publique pour séjour irrégulier qu'envers les étrangers ayant également commis une autre infraction et de classer sans suite les autres procédures afin, notamment, de laisser prospérer la procédure administrative d'éloignement du territoire ».

8 Quelques jours seulement avant que la CJUE rende l'arrêt El Dridi, le Parlement français adoptait une importante réforme du régime de la garde à vue, sous la contrainte de plusieurs décisions de la Cour Européenne des Droits de l'Homme (CEDH) ${ }^{6}$, auxquelles elle avait d'abord longtemps résisté. Pour tenter de limiter l'usage excessif de cette mesure de contrainte, la nouvelle loi généralisait notamment la règle selon laquelle elle ne peut être appliquée qu'à des personnes soupçonnées d'avoir commis une infraction 
punie, au moins, d'une peine d'emprisonnement. Certes, tel était bien le cas du séjour irrégulier, qui était puni d'une peine d'un an d'emprisonnement par l'article L 621-1 du CESEDA. Mais deux semaines seulement après la publication de la loi du 14 avril 2011, l'arrêt $E l$ Dridi énonçait que les législations nationales ne peuvent pas prévoir des peines d'emprisonnement pour sanctionner le fait de demeurer sur le territoire d'un État membre en violation d'un ordre de quitter ce territoire. Il s'en déduisait alors, naturellement, que la garde à vue ne pouvait plus être utilisée pour retenir les personnes soupçonnées d'avoir commis l'infraction de séjour irrégulier: la peine d'emprisonnement dont elle était punie était devenue illégale au regard du droit de l'Union et elle ne pouvait donc justifier, en aucun cas, l'utilisation de cette mesure de contrainte.

9 Dans ce nouveau contexte, l'étranger dont le séjour irrégulier était découvert à l'occasion d'un contrôle ne pouvait plus être retenu, en principe, que pendant les quatre heures, au maximum, qu'autorise le Code de procédure pénale pour procéder à une vérification d'identité. Or ce délai devenait souvent très insuffisant pour permettre à l'administration de prendre les décisions et les dispositions pratiques nécessaires pour engager la procédure d'éloignement. En remettant en cause le processus juridique qui conduisait les étrangers des locaux de garde à vue vers les centres de rétention administrative, puis vers les salles d'embarquement, la combinaison de l'arrêt $E l$ Dridi et du régime de la garde à vue a donc produit un effet inattendu: elle a privé l'administration d'une étape clé du processus d'éloignement.

Pour tenter d'échapper aux conséquences - pourtant tout à fait évidentes - de cet arrêt, les autorités administratives se sont immédiatement et exclusivement montré préoccupées d'assurer la survie artificielle de ce dispositif, reposant sur l'articulation opportuniste et confortable entre garde à vue judiciaire et rétention administrative. Les ministères de l'Intérieur et de la Justice ont ainsi déployé, avec ténacité, des trésors d'argumentation et d'imagination pour tenter d'échapper à la nécessité de repenser les fondements juridiques des procédures d'éloignement.

11 Cette attitude défensive constitue, sans aucun doute, la seconde explication de la durée et de la complexité des effets produits par les arrêts El Dridi et Achughbabian. En privilégiant des interprétations tronquées, erronées et partiales de ces décisions, ces autorités ont en effet créé une telle confusion dans l'esprit de certains des magistrats chargés de statuer sur la régularité des procédures d'éloignement qu'il s'en est suivi une longue cacophonie judiciaire. Non seulement il n'a été mis fin à cette confusion, par un arrêt de la Cour de cassation, que plus d'un an après l'arrêt El Dridi mais, plus surprenant encore, d'ultimes résistances aux conséquences de cet arrêt se sont encore manifestées ensuite et ce, jusqu'à ce que le législateur réintroduise finalement, dans notre législation, un ersatz de garde à vue.

De toute évidence, cette obstination des autorités trouve un début d'explication dans le choix fait par le gouvernement de l'époque d'afficher les résultats concrets de sa politique d'éloignement des étrangers en situation irrégulière. Approchant d'une échéance électorale majeure, il espérait sans doute regagner ainsi les faveurs d'une opinion publique - supposée acquise aux discours d'exclusion et de repli national - en se montrant efficace dans la mise en œuvre de cette politique. Pourtant, la volonté d'atteindre des objectifs tournant autour de 30000 mesures d'éloignement par an ne peut expliquer à elle seule l'étonnante application avec laquelle des ministres, préfets, fonctionnaires, directeurs d'administrations, chefs de juridictions, procureurs et... 
juges, ont tenté de contourner les difficultés juridiques qui contrariaient soudain la mise en œuvre de cette politique volontariste. Faute de pouvoir identifier et analyser tous les ressorts de l'espèce de consensus tacite qui s'est formé pour sauvegarder un schéma procédural de l'éloignement fortement fragilisé, on tentera au moins de mettre en évidence les manifestations successives de cette résistance organisée.

\section{L'arrêt El Dridi : les ministres donnent le ton} qui l'avait immédiatement interrogé sur ce point ${ }^{9}$, le garde des Sceaux s'est néanmoins trouvé contraint de prendre très rapidement position par une circulaire du 12 mai 2011 (soit quinze jours seulement après l'arrêt $E l$ Dridi) adressée aux procureurs généraux « compte tenu notamment des divergences d'interprétation observées ces derniers jours entre les diverses cours d'appel quant à la portée exacte de cet arrêt de la CJUE». Adoptant une stratégie moins grossière que celle du ministre de l'Intérieur, il admettait que le fait de se soustraire à une mesure d'éloignement ne pourrait plus donner lieu à une peine d'emprisonnement. Mieux, il en tirait la conséquence nécessaire qu'il n'était plus possible, non plus, de placer en garde à vue celui qui était soupçonné de s'être soustrait à une telle mesure. En revanche, ayant ainsi concédé le plus évident, il distinguait nettement la situation de l'étranger en situation irrégulière qui n'avait pas encore fait l'objet d'une décision d'éloignement - et ne pouvait donc pas s'être soustrait à une telle mesure - considérant arbitrairement que celle-ci restait hors d'atteinte de l'arrêt $E l$ Dridi.

15 L'erreur était pourtant non seulement manifeste mais double. Erreur de droit, d'abord, parce que la directive (article 6) impose d'engager la procédure d'éloignement à l'égard de tout étranger en situation irrégulière sans distinction et que "l'infliction » d'une peine d'emprisonnement fait toujours obstacle à l'éloignement, quel que soit le cas de figure. Or, c'est précisément cet obstacle que la CJUE a voulu écarter avec l'arrêt $E l$ Dridi. Erreur de simple bon sens, ensuite, puisque ce distinguo aboutit à infliger un peine d'emprisonnement à l'étranger qui se trouve dans la situation la moins "grave " - un séjour irrégulier "simple» qui n'a encore donné lieu à aucune décision d'éloignement - alors que celui qui s'est soustrait à une telle décision n'encourrait pas cette peine.

Sans se soucier de ces approximations et contradictions, la circulaire demandait pourtant aux procureurs généraux de «veiller à ce que soient systématiquement frappées 
d'appel les décisions de refus de prolongation des mesures de rétention administrative fondées sur l'inconventionnalité alléguée de l'article L 621-1 du CESEDA » ajoutant : " en cas de rejet de l'appel vous formerez un pourvoi en cassation ». Il n'était donc pas question que s'exprime la moindre velléité d'appliquer le droit de l'Union, dont une interprétation plus rigoureuse devait pourtant conduire à écarter les dispositions assorties de peines d'emprisonnement et, avec elles, celles relatives à la garde à vue. Mais à l'inverse du résultat recherché, ces instructions n'ont fait que multiplier les divergences d'interprétation qu'elles cherchaient à atténuer. De nombreux juges des libertés et de la détention (JLD) et cours d'appel ne se sont pas laissé abuser, en effet, par l'analyse du ministère de la Justice. Ils refusaient donc de prolonger les rétentions précédées d'une garde à vue fondée sur le séjour irrégulier et ce, même lorsque l'étranger ne s'était pas soustrait à une mesure d'éloignement préexistante. Si bien qu'après de nombreux mois de flottements, la cour d'appel de Paris a décidé de saisir à son tour la CJUE d'une question préjudicielle par une décision du 29 juin $2011^{10}$. La question était ainsi formulée : «La directive 2008/115/CE du 16 décembre 2008 s'oppose-t-elle à une réglementation nationale, telle que l'article L621-1 du CESEDA prévoyant l'infliction d'une peine d'emprisonnement à un ressortissant d'un pays tiers au seul motif de l'irrégularité de son entrée ou de son séjour sur le territoire national ?».

\section{L'arrêt Achughbabian : les ministres s'entêtent !}

17 On sait quelle a été la réponse de la grande chambre : " la directive doit être interprétée en ce sens qu'elle s'oppose à une réglementation réprimant le séjour irrégulier par des sanctions pénales pour autant que celle-ci permet l'emprisonnement [d'un étranger en situation irrégulière qui] n'a pas été soumis aux mesures coercitives visées à l'article 8 de cette directive... ». Pourtant, les ministres de l'Intérieur et de la Justice déclaraient dès le lendemain cette fois avec une belle unanimité - que « la CJUE confirme que le recours à la garde à vue en matière de séjour irrégulier est compatible avec le droit communautaire». Et, de nouveau, le résultat ne s'est pas fait attendre: dans les jours qui ont suivi les JLD et les cours d'appel, dont celle de Paris, ont continué de rendre des décisions contradictoires, certaines se pliant à la jurisprudence Achughbabian, d'autres suivant les ministres dans leur attitude de résistance, avec cette triste conséquence que le sort des étrangers continuait d'être soumis au seul aléa des options jurisprudentielles de la juridiction saisie.

Une nouvelle circulaire a donc été publiée le 13 décembre 2011 par le ministère de la Justice. Elle tentait astucieusement - mais abusivement- de tirer parti des «considérant» 30 et 32 de l'arrêt selon lesquels la directive «ne s'oppose pas à la détention d'un ressortissant d'un pays tiers - ou à une privation de liberté telle qu'une garde à vue - en vue de la détermination du caractère régulier ou non du séjour de celui-ci. » Mais pour en déduire que la garde à vue restait autorisée en matière de séjour irrégulier, le ministre « oubliait » tout simplement que si la directive ne s'oppose pas, en effet, à une privation de liberté "telle qu'une garde à vue », en revanche la loi française, elle, s'y opposait totalement. La circulaire « escamotait » en effet le nouvel article 62-2 du Code de procédure pénale qui dispose pourtant que la garde à vue ne peut être utilisée que si l'intéressé encourt une peine d'emprisonnement. Or c'est précisément ce que la directive interdit en matière de séjour irrégulier, comme la Cour venait de l'affirmer à 
nouveau solennellement (à moins, seulement, que l'intéressé ait déjà été soumis à l'ensemble des mesures coercitives prévues par la directive) ${ }^{11}$.

Négligeant totalement cette conséquence de l'arrêt Achughbabian, la circulaire affirme : "Ce n'est qu'au stade de l'engagement des poursuites pénales contre l'étranger en situation irrégulière au titre de l'article L 621-1, et non lors du placement en garde à vue, que l'arrêt de la Cour serait susceptible de produire ses effets ». Le subterfuge était d'autant plus grossier que la Cour avait pris soin de préciser, en réponse précisément à un argument du gouvernement français, que la réglementation prévoyant l'infliction d'une peine d'emprisonnement restait incompatible avec la directive même en présence de circulaires prévoyant d'abandonner les poursuites pénales dès la fin de la garde à vue. Il suffit en effet que cette réglementation soit seulement "susceptible de conduire à une condamnation " pour qu'elle soit " condamnée " par la directive. Il suffisait donc que l'article L 621-1 reste potentiellement applicable - même s'il n'était pas appliqué de facto - pour qu'il soit, comme le dit le considérant 39 de l'arrêt Achughbabian " susceptible de faire échec à l'application des normes et des procédures communes établies par la directive [...] portant ainsi atteinte à l'effet utile de la directive ».

Mais le ministre de la Justice ne s'embarrassait pas de ces détails: la circulaire se terminait par la même instruction adressée aux procureurs généraux de «veiller à ce que soient systématiquement frappées d'appel les décisions fondées sur l'irrégularité alléguée des placements en garde à vue opérés sur la base de l'article L 621-1 du CESEDA » et de former un pourvoi en cassation en cas de rejet de l'appel.

21 Pourtant, ce n'est pas sur un pourvoi du ministère public que la Cour de cassation s'est finalement prononcée, plus de six mois après cette circulaire et dans une confusion jurisprudentielle persistante. Elle l'a fait sur les pourvois formés par des étrangers contre des décisions autorisant la prolongation de leur rétention alors qu'ils avaient été initialement placés en garde à vue. Mais avant que la Cour de cassation se prononce, le Conseil constitutionnel avait lui-même été saisi d'une question prioritaire de constitutionnalité (QPC) visant l'article L 621-1 du CESEDA. Cette saisine a constitué une occasion supplémentaire de vérifier le consensus qui s'était formé pour ne pas remettre en cause la pénalisation du séjour irrégulier et, avec elle, le dispositif d'éloignement reposant sur la mobilisation de la garde à vue.

\section{Le Conseil constitutionnel se dérobe}

Le Conseil constitutionnel a donc été saisi le 23 novembre 2011 de la question de la conformité de l'article L 621-1 du CESEDA aux droits et libertés que garantissent la Constitution et plusieurs textes fondamentaux intégrés dans le bloc de constitutionnalité. L'auteur de la question, M. Mohamed AkliB, et plusieurs associations intervenues pour le soutenir faisaient valoir que la peine d'un an d'emprisonnement sanctionnant le séjour irrégulier n'était pas conforme à l'article 8 de la Déclaration des droits de l'homme et du citoyen de 1789, selon lequel «la loi ne doit établir que des peines strictement et évidemment nécessaires ».

Le raisonnement était à la fois simple et robuste : primo, la Cour de justice de l'Union européenne a constaté que l'infliction d'une peine d'emprisonnement pour le simple fait d'être en situation de séjour irrégulier est susceptible de faire obstacle à la mise en œuvre effective de la procédure d'éloignement, à laquelle une priorité absolue doit être donnée ; secundo, elle a dit pour droit que les États membres doivent s'abstenir de toute 
mesure susceptible de mettre en péril la réalisation des objectifs de la directive ; tertio, comment l'infliction d'une peine d'emprisonnement au cours de la procédure d'éloignement pourrait-elle être nécessaire alors qu'elle est au contraire interdite par le droit de l'Union?

Autrement dit, si la directive empêche l'application de cette peine pendant la procédure de retour, l'incrimination ne répond plus à aucune nécessité dès que la situation de séjour irrégulier est mise en évidence et jusqu'à ce que toutes les mesures coercitives prévues par la directive aient été mises en œuvre. L'article L 621-1 ne sera donc conforme à l'article 8 de la Déclaration des droits de l'homme que lorsque son application aura été expressément limitée à la situation de l'étranger qui a déjà fait l'objet de toutes ces mesures coercitives. Cette argumentation était d'ailleurs renforcée par le rappel des instructions qui avaient été données aux procureurs de la République de ne pas poursuivre les infractions de simple séjour irrégulier "afin, notamment, de laisser prospérer la procédure administrative d'éloignement du territoire » : quelle meilleure preuve, en effet, que ces poursuites n'étaient plus nécessaires à partir du moment où l'éloignement était devenu l'objectif prioritaire, pour ne pas dire exclusif?

Par une décision $\mathrm{n}^{\circ}$ 2011-217 QPC du 3 février 2012 ${ }^{12}$ déclarant l'article L 621-1 conforme à la Constitution, le Conseil a en réalité esquivé le débat. Refusant d'affronter le raisonnement qui lui était proposé, il s'est contenté de reprendre sa jurisprudence habituelle selon laquelle « un grief tiré du défaut de compatibilité d'une disposition législative aux engagements internationaux et européens de la France ne saurait être regardé comme un grief d'inconstitutionnalité », ajoutant que "par suite, il n'appartient pas au Conseil constitutionnel... d'examiner la compatibilité des dispositions contestées avec les traités ou le droit de l'Union européenne, l'examen d'un tel grief relevant de la compétence des juridictions administratives et judiciaires ». Brillant par son courage, le Conseil a ainsi trouvé refuge dans la distinction entre le contrôle de constitutionnalité, qui lui revient de droit, et le contrôle de conformité de la loi au droit de l'Union qu'il attribue aux seules juridictions « ordinaires ».

Pourtant, ni le requérant ni les associations intervenantes ne demandaient au Conseil constitutionnel de contrôler la conformité de l'article L 621-1 au droit de l'Union et de revenir ainsi sur la jurisprudence par laquelle il avait déjà délimité son propre champ de compétence. Ils lui demandaient bien, au contraire, d'évaluer la conformité de cet article au principe de nécessité des peines. Mais ils lui demandaient de le faire en tirant les conséquences des arrêts de la Cour de justice de l'Union européenne dans la mesure où l'interdiction d'infliger une peine d'emprisonnement, qui se déduit de ces arrêts, modifiait nécessairement l'appréciation de cette nécessité. La Cour de justice ayant déjà dit pour droit que l'emprisonnement était incompatible avec la directive tant que la procédure d'éloignement n'a pas été menée à son terme, ce principe était ainsi déjà intégré dans notre droit interne. Le Conseil pouvait donc lui-même en déduire que cette peine n'était, ipso facto, plus nécessaire au sens de l'article 8 de la Déclaration des droits de l'homme et du citoyen, sans procéder pour autant à un contrôle de conformité de l'article L 621-1 au droit de l'Union.

Confirmant ainsi son refus d'invalider le dispositif de pénalisation du séjour irrégulier, le Conseil a d'ailleurs également rejeté le second argument invoqué par le requérant selon lequel les peines de un an d'emprisonnement et de 3750 euros d'amende prévues à l'article L 621-1 du CESEDA étaient sans proportion avec la gravité relative du délit de séjour irrégulier. Sur ce point, il s'est contenté d'affirmer "qu'eu égard à la nature de 
l'incrimination pour laquelle elles sont instituées, les peines ainsi fixées ne sont pas manifestement disproportionnées ».

Le Conseil constitutionnel ayant ainsi endossé les habits de Ponce Pilate en renvoyant le sort de l'article L 621-1 à l'appréciation du juge judiciaire, tous les espoirs des défenseurs des étrangers se sont alors tournés vers la Cour de cassation. Certes, ils n'ont pas été déçus ... mais c'était sans compter sur de nouvelles capacités de résistance qui ont néanmoins continué de se manifester de toutes parts.

\section{La Cour de cassation cède}

Alors que de nombreuses juridictions avaient immédiatement tiré les conséquences de l'arrêt $E l$ Dridi en annulant les procédures d'éloignement qui avaient débuté par une garde à vue, d'autres avaient au contraire tenté de passer outre, évidemment encouragées par les déclarations du ministre de l'Intérieur et la première circulaire du ministre de la Justice. La première chambre civile de la Cour de cassation a donc été saisie, dès le mois de mai 2011, de plusieurs pourvois formés contre des arrêts de Cours d'appel ayant autorisé la prolongation de rétentions précédées d'une garde à vue. Bien entendu, ces pourvois posaient clairement la question de la nullité de ces gardes à vue résultant de l'impossibilité de punir le séjour irrégulier d'une peine d'emprisonnement. Après une gestation de près d'un an, la première chambre civile - naturellement compétente en matière de procédures d'éloignement - a choisi de saisir la chambre criminelle de la même Cour, compétente en matière de procédure pénale, d'une demande d'avis ainsi formulée : "À la lumière des arrêts de la Cour de justice de l'Union européenne du 28 avril et du 6 décembre 2011 [ainsi que des dispositions en vigueur avant et après la réforme de la garde à vue] un ressortissant d'un État tiers à l'Union européenne peut-il être placé en garde à vue sur le fondement du seul article L 621-1 du CESEDA ?»

Bien que l'avocat général ait conclu, par un raisonnement assez obscur, que cet article "n'est pas contraire à la directive retour » et qu' « il peut donc être le support d'une garde à vue", l'avis rendu le 5 juin 2012 par la chambre criminelle $^{13}$ a été particulièrement net et tranché. Elle commence par rappeler que la garde à vue «doit obéir à l'un des objectifs nécessaires à la conduite de la procédure pénale engagée ", autre façon de dire qu'elle ne peut pas être instrumentalisée au bénéfice d'une procédure administrative. Elle affirme ensuite qu' «à la suite de l'entrée en application de la directive du 16 décembre 2008 [...] telle qu'interprétée par la Cour de justice de l'Union européenne, le ressortissant d'un État tiers mis en cause pour le seul délit prévu par l'article L 621-1 n'encourt pas l'emprisonnement lorsqu'il n'a pas été soumis préalablement aux mesures coercitives visées à l'article 8 de ladite directive " et qu'« il ne peut donc être placé en garde à vue à l'occasion d'une procédure diligentée de ce seul chef ».

31 Tous les commentateurs s'accordaient pour considérer que la première chambre civile pourrait difficilement s'écarter de cet avis particulièrement péremptoire, mais la résistance n'en a pas moins continué, dans les semaines suivantes, au sein de nombreuses juridictions. Ainsi le procureur de la République de Paris faisait-il savoir à ses substituts, quelques jours après cet avis, qu'il n'entendait rien changer aux pratiques antérieures et qu'il convenait donc de continuer à autoriser les officiers de police judiciaire à utiliser la garde à vue. Il faut préciser, pour mieux comprendre cet acharnement, qu'au moment où la circulaire qui tentait de contredire l'arrêt El Dridi 
était signée et diffusée l'intéressé n'était pas encore procureur de la République de Paris, mais qu'il était en revanche... directeur du cabinet du ministre de la justice !

Il a donc fallu attendre un mois encore avant que les arrêts rendus le 5 juillet 2012 par la première chambre civile ${ }^{14}$ confirment définitivement l'avis de la chambre criminelle. Avec, toutefois, une petite nuance "en retrait »: ils précisent que la garde à vue reste possible dès lors que l'étranger a été soumis à «l'une » des mesures coercitives prévues par la directive alors que l'arrêt Achughbabian exige clairement l'échec de l'ensemble de ces mesures coercitives pour que l'emprisonnement devienne possible.

À cette petite réserve près, il était permis de penser que les exigences de la directive «retour » avaient enfin triomphé. Les conséquences, inéluctables, de la jurisprudence de la CJUE étaient enfin admises et l'inadaptation du droit français de la garde à vue à la réalisation des objectifs de la directive était enfin reconnue et consacrée. La victoire avait cependant un goût amer ${ }^{15}: \mathrm{du}$ fait de tous les obstacles que cette solution de bon sens avait rencontrés, des centaines d'étrangers, sans doute, avaient été expulsés au terme d'une procédure illégale.

34 Contraints de renier les instructions précédemment données aux procureurs généraux et procureurs de la République par leurs prédécesseurs, les directeurs des affaires criminelles et des affaires civiles du ministère de la justice diffusaient, dès le 6 juillet, une nouvelle circulaire tirant les conséquences des arrêts de la Cour de cassation. Ils reconnaissaient ainsi, implicitement mais nécessairement, qu'ils avaient encouragé et validé pendant plus d'un an des procédures attentatoires aux libertés individuelles et radicalement nulles. De même, le ministre de l'Intérieur diffusait parallèlement les instructions nécessaires pour que les services de police s'adaptent au nouveau contexte juridique créé par les arrêts du 5 juillet dans lequel les procédures d'éloignement des étrangers en situation irrégulière devaient dorénavant s'inscrire.

Deux considérations, au moins, permettaient d'attendre que ces circulaire et instructions traduisent une capitulation totale et inconditionnelle: le caractère particulièrement net de la solution dégagée par la Cour de cassation, d'une part et l'alternance politique qui venait de se produire, d'autre part. Il était en effet permis d'espérer qu'elle favoriserait un changement de perspective et d'approche dans le traitement de l'ensemble des questions relatives aux droits des étrangers. C'est pourtant la volonté affirmée de ne surtout pas baisser la garde dans la mise en œuvre d'une politique efficace d'éloignement des étrangers en situation irrégulière qui a manifestement prévalu dans les initiatives prises, chacun dans son domaine de compétence, par les deux ministères.

$36 \mathrm{Au}$ demeurant, la circulaire du ministre de l'Intérieur était diffusée dans l'attente, annonçait-il déjà, "de nouvelles règles, prenant en compte les impératifs constitutionnels et européens, [qui] vont venir garantir un équilibre indispensable entre le respect des libertés individuelles et les exigences de maitrise des flux migratoires »: que la maîtrise des flux migratoires - perspective aussi vaine qu'incantatoire - soit érigée en "exigence» pouvait déjà faire douter d'une réelle volonté de changement de politique à l'égard des étrangers. 


\section{Le ministère de la Justice encourage les expédients}

37 C'est du bout des lèvres que la circulaire diffusée par le ministère de la Justice s'incline devant les arrêts de la Cour de cassation en demandant aux procureurs d'« inviter les officiers de police judiciaire à éviter de recourir désormais à une mesure de garde à vue du seul chef de séjour irrégulier ». Il est, en effet, pour le moins souhaitable d' «éviter» de faire usage d'une procédure nulle car successivement déclarée contraire à notre droit par les plus hautes autorités judiciaires européennes et nationales : seuls des esprits chagrins pourraient regretter de voir donner ces instructions en termes aussi mesurés...

Cette retenue prêterait presque à sourire si les auteurs de la circulaire ne s'étaient pas, ensuite, aventurés sur un terrain beaucoup plus contestable. Ils ont en effet entendu récapituler les autres dispositions de procédure pénale qui restaient mobilisables par les enquêteurs pour retenir les étrangers pendant le temps nécessaire à l'engagement de la procédure administrative d'éloignement. Car tel était bien, manifestement, l'enjeu auquel ils entendaient répondre : trouver coûte que coûte un substitut à la garde à vue offrant les mêmes avantages en termes de « confort » et d'efficacité administrative.

La circulaire rappelle donc d'abord, sans surprise, que l'étranger qui refuse ou est dans l'impossibilité de justifier de son identité peut être retenu pendant quatre heures pour vérification d'identité et que «les services de police ou de gendarmerie disposent alors de la faculté d'informer les services de la préfecture ». Mais, elle livre ensuite une lecture tronquée et déformée des dispositions des articles 62, 73 et 78 du Code de procédure pénale relatives à l'audition sans garde à vue, couramment dénommée "audition libre ». Il s'agit d'un régime d'audition réservé aux seules personnes à l'encontre desquelles il n'existe encore aucune raison de soupçonner qu'elles ont commis une infraction ou qui ont été appréhendées et conduites devant l'officier de police judiciaire par un simple particulier. Bien que ces conditions ne soient pas réunies lorsque le séjour irrégulier d'un étranger est découvert à l'occasion d'un contrôle d'identité, la circulaire précise que l'utilisation de cette procédure «est juridiquement concevable... si la personne n'a pas été conduite par la force publique sous la contrainte devant un officier de police judiciaire ». Le mode d'emploi était ensuite précisé : l'officier de police judiciaire doit demander à l'étranger de confirmer sur procès-verbal qu'il a suivi les agents de la force publique de son plein gré et il doit être informé qu'il peut quitter les locaux de police à tout moment.

Comme par l'effet du hasard, les étrangers ont commencé à se montrer soudainement très dociles dans les semaines suivantes: les procès-verbaux constataient miraculeusement leur entier accord pour suivre les policiers et rester tranquillement dans les locaux de police en attendant que les préfets délivrent les décisions d'éloignement mettant brutalement fin à la vie familiale et/ou professionnelle qu'ils avaient organisée en France. Et il s'est malheureusement trouvé de nombreux juges pour admettre la fiction d'un consentement qui n'avait manifestement pu être donné s'il l'avait réellement été - en toute connaissance de cause. Mais là n'est pas encore le plus grave. Car une analyse rigoureuse des dispositions du Code de procédure pénale régissant l'audition libre démontrait clairement qu'elles ne peuvent être utilisées en matière de recherche d'infractions de séjour irrégulier, ce que le garde des Sceaux ne pouvait évidemment ignorer ${ }^{16}$. Pourtant, aucun rectificatif n'a été publié et, comme il fallait s'y attendre, la plus grande confusion a de nouveau régné entre les différentes 
juridictions chargées de statuer sur la régularité des procédures d'audition libre qui se multipliaient curieusement.

41 Si plusieurs de ces juridictions ont fini par reconnaître que l'audition libre se heurtait à une impossibilité juridique incontournable - malgré la suggestion d'y recourir subtilement distillée par le ministère - certaines d'entre elles n'ont pas pour autant désarmé ni renoncé à favoriser la mise en œuvre des procédures d'éloignement par tous les moyens possibles. C'est notamment le cas de la Cour d'appel de Paris, qui n'a annulé des procédures engagées sous le régime de l'audition libre qu'après avoir trouvé une énième solution pour contourner à nouveau les difficultés pratiques résultant de l'abandon de la garde à vue. Se référant à deux anciens arrêts de la Cour de cassation des 14 juin 2005 et 25 novembre $2009^{17}$, elle a en effet considéré qu'aucune mesure d'enquête judiciaire n'étant nécessaire lorsque l'irrégularité du séjour a été constatée dès le contrôle d'identité (par exemple après consultation du fichier national des étrangers), l'étranger pouvait alors être retenu dans les locaux de police dans le cadre d'une procédure purement administrative destinée à permettre au préfet de le placer en rétention.

Autrement dit, pour pallier l'absence de garde à vue la cour d'appel choisissait finalement la solution la plus expéditive : la retenue de l'étranger était suffisamment justifiée par la nécessité de le remettre à l'autorité administrative! Quitte à fermer les yeux sur ce qui fait la monstruosité de cette ultime manœuvre de sauvetage des procédures d'éloignement: entre l'interpellation de l'étranger et la délivrance de l'arrêté préfectoral le plaçant en rétention, celui-ci est arbitrairement privé de liberté puisqu'il n'existe aucun titre, ni judiciaire (pas de garde à vue, ni d'audition « libre ») ni administratif (l'arrêté n'a pas encore été pris), fondant juridiquement la contrainte qui s'exerce sur lui.

43 La Cour d'appel de Paris n'a pas été la seule à recourir à des expédients de plus en plus sommaires pour passer outre les principes qui l'empêchaient de valider les procédures d'éloignement. Paraissant regretter d'avoir dû suivre l'avis de la chambre criminelle, la première chambre civile de la Cour de cassation, elle-même, rendait le 21 novembre $2012^{18}$ un arrêt stupéfiant, cassant une décision ayant logiquement constaté qu'aucun cadre légal n'était de nature à justifier la privation de liberté de l'étranger entre son interpellation et son placement en rétention. Pour motiver cette cassation la Cour se borne à constater que "l'intéressé a été retenu au commissariat de police en raison de sa situation irrégulière en France et qu'il en a été informé dans le plus court délai et dans une langue qu'il comprenait au cours d'une procédure qui répondait aux exigences posées par l'article 62 du Code de procédure pénale ». Or il faut préciser que cet article 62 est celui qui permet l'audition libre des personnes "à l'encontre desquelles il n'existe aucune raison plausible de soupçonner qu'elles ont commis ou tenté de commettre une infraction »... La Cour de cassation justifie donc la retenue sous la contrainte d'un étranger dont la situation irrégulière est avérée en considérant qu'elle est conforme aux dispositions régissant l'audition sans contrainte des personnes qui ne sont soupçonnées d'aucune infraction! Et comme la Cour d'appel de Paris, elle ignore volontairement que cette retenue a été imposée sans être fondée sur aucun titre juridique...

Il était peut-être souhaitable, finalement, que le législateur intervienne pour mettre fin à de tels errements... 


\section{Le ministre de l'Intérieur organise la continuité}

L'intervention du législateur était en réalité inévitable pour mettre les dispositions du CESEDA relatives au séjour irrégulier en conformité avec le droit de l'Union: l'article L 621-1 ayant été déclaré incompatible avec la directive du 16 décembre 2008, il fallait en tirer les conséquences. C'est ce que fait la loi nº 2012-1560 du 31 décembre 2012 en l'abrogeant purement et simplement. Bien qu'attendue par les associations de défense des étrangers, cette dépénalisation du séjour irrégulier ne peut pas être regardée, pour autant, comme le signe d'un profond renversement des principes qui guident le gouvernement dans son action en direction des étrangers. Preuve que l'obsession de la pénalisation du séjour irrégulier reste tenace, la loi maintient le délit d'entrée irrégulière et, surtout, elle crée parallèlement un nouveau délit de « résistance passive » sanctionnant l'étranger qui se maintient sur le territoire, malgré un ordre de le quitter, "après avoir fait l'objet d'une mesure régulière de placement en rétention ou d'assignation à résidence».

$\mathrm{Au}$ demeurant, pour présenter et faire adopter en urgence - selon la procédure accélérée ne permettant qu'une seule lecture devant chaque assemblée du Parlement son projet de loi "relatif à la retenue pour vérification du droit au séjour ", le ministre de l'intérieur n'a pas invoqué l'urgente nécessité de faire cesser une répression inutile et injustifiée. Il a en revanche invoqué l'existence d'un "vide juridique " résultant de l'impossibilité de recourir à la garde à vue pour mener à bien les procédures d'éloignement. Chacun a bien compris, pourtant, que s'il y avait un vide il n'était pas juridique mais «technique » et qu'il s'agissait seulement d'offrir assez de temps aux services des préfectures pour réagir à l'interpellation des étrangers en situation irrégulière. La culture de l'efficacité et du chiffre perdurait et exigeait que l'on trouve d'urgence un substitut à la garde à vue.

Même pour satisfaire les objectifs "productivistes » d'interpellation et d'éloignement des étrangers en situation irrégulière que le nouveau gouvernement disait vouloir poursuivre "avec fermeté et humanité », l'intervention du législateur n'était d'ailleurs sans doute pas nécessaire. Les observations des associations intervenant dans les centres de rétention avaient montré, en effet, que le nombre d'étrangers qui y étaient placés n'avait pas baissé significativement au premier semestre 2012 et la préfecture de police de Paris avait d'ailleurs pu maintenir le nombre de reconduites à la frontière au niveau «habituel». Beaucoup d'associations de défense des étrangers ont au demeurant plaidé pour le maintien du statu quo résultant des arrêts de la Cour de cassation, faisant valoir à juste titre qu'une initiative législative créant un dispositif dérogatoire d'enfermement était non seulement inutile mais politiquement inopportune compte tenu du message hostile aux étrangers qu'elle véhiculait. Telle n'a pas été l'option retenue, le ministre de l'Intérieur ayant annoncé dès le 28 juin 2012 son intention de remplacer la garde à vue par "un outil législatif qui permette de s'assurer que les étrangers en situation irrégulière regagnent leur pays d'origine" pour éviter de "nous retrouver dans une situation où il n'y aurait plus de reconduites à la frontière».

La loi fournit donc ce nouvel outil en permettant aux officiers de police judiciaire de "retenir» un étranger dans un local de police "pour le temps strictement exigé par l'examen de son droit de circulation ou de séjour et, le cas échéant, le prononcé et la notification des décisions administratives applicables ", étant précisé que la durée de cette retenue ne peut excéder seize heures. Cette retenue répond donc à une finalité purement 
administrative - la détection des étrangers en situation irrégulière et leur reconduite à la frontière - ce qui en fait la différence essentielle avec la garde à vue, à finalité pénale. Elle est néanmoins placée sous le contrôle (théorique et illusoire compte tenu de ses modalités) du procureur de la République, dont notre Constitution fait une "autorité judiciaire" malgré les réserves de la CEDH sur ce point ${ }^{19}$. Elle emprunte d'ailleurs très largement au régime et aux modalités de la garde à vue, dont elle constitue une duplication quasi-parfaite. Si bien que la nature juridique administrative, par son objet, ou judiciaire, par son régime - de cette mesure privative de liberté reste finalement assez ambiguë, ce qui peut susciter des interrogations quant à la nature et au régime des recours que sa mise en œuvre pourra justifier. Sur cette question au moins, il n'est pas certain que l'application de la loi nouvelle permette d'échapper à des débats jurisprudentiels qui pourraient être aussi intenses que ceux qui ont précédé son adoption.

49 Il y a un paradoxe dans cette résistance organisée à la jurisprudence de la CJUE : les arrêts $E l$ Dridi et Achughbabian ne visent en effet qu'à satisfaire un objectif d'éloignement prioritaire des étrangers en situation irrégulière imposé par la directive qui coïncide parfaitement, en réalité, avec ceux de nos gouvernements successifs. Si ces arrêts ont néanmoins rencontré une résistance farouche, c'est donc exclusivement parce qu'en interdisant l'emprisonnement de ces étrangers ils empêchaient de faire de la garde à vue le « support technique » des procédures d'éloignement ${ }^{20}$.

Certes, l'arrêt El Dridi pouvait encore laisser penser que si la CJUE était préoccupée de donner son plein effet à la directive, elle était également très attentive à ce que les États membres accordent la plus grande attention au respect des droits fondamentaux et des principes de gradation et de proportionnalité dans l'usage des mesures de contrainte. De ce point de vue, la dépénalisation du séjour irrégulier pouvait peut-être encore apparaître comme la conséquence commune de l'une et l'autre de ces deux préoccupations. Mais l'arrêt Sagor du 6 décembre 2012 (C-430/11) ruine définitivement (un an jour pour jour après l'arrêt Achughbabian) cette interprétation " angélique » de la position des juges de Luxembourg qui les imaginait tout aussi soucieux du respect des droits fondamentaux que de l'efficacité de la politique d'éloignement. La Cour y déclare que la directive du 16 décembre 2008 "s'oppose à une réglementation d'un État membre qui permet de réprimer le séjour irrégulier par une peine d'assignation à résidence sans garantir que l'exécution de cette peine doive prendre fin dès que le transfert physique de l'intéressé hors dudit État membre est possible». Mais pour arriver à cette conclusion, elle abandonne ici toute référence aux droits fondamentaux et réaffirme sans nuance aucune la nécessité de faire prévaloir l'éloignement des étrangers sur toute autre considération. 


\section{NOTES}

1. CJUE, 28 avr. 2011, C-61/11, Hassen El Dridi alias Soufi Karim.

2. «La pénalisation du séjour irrégulier est contraire aux objectifs de droit de l'Union européenne » par Marie-Laure Basilien-Gainche, http://combatsdroitshomme.blog.lemonde.fr/ ; « La directive "retour" au secours des étrangers? ", Fabienne Kauff-Gazin, Lexis Nexis Europe n 6 , juin 2011, étude 7.

3. Directive du Parlement européen et du Conseil relative aux normes et procédures communes applicables dans les États membres au retour des ressortissants de pays tiers en séjour irrégulier. 4. CJUE, 6 déc. 2011, C-329/11, Alexandre Achughbabain c/ Préfet du Val de Marne ; Serge Slama, «La Cour de justice consacre l'ubiquité du délit de séjour irrégulier, à la fois conforme et non conforme au droit de l'Union européenne selon la phase de la procédure de retour» in Lettre "Actualités DroitsLibertés " du CREDOF, 7 décembre 2011; "Directive retour et peine d'emprisonnement: l'article L 621-1 du CESEDA épinglé », C. Fleuriot, Dalloz Actualité, 7 décembre 2011 (http:// www.dalloz-actualite.fr); P. Henriot, «Garde à vue et séjour irrégulier: les enseignements de l'arrêt Achughbabian sont limpides ", Gaz. Pal. 12 au 14 février 2012, p. 17.

5. Loi $n^{\circ}$ 2012-1560 du 31 décembre 2012 « relative à la retenue pour vérification du droit au séjour et modifiant le délit d'aide au séjour irrégulier pour en exclure les actions humanitaires désintéressées ».

6. Notamment: CEDH Salduz c/ Turquie, 27 nov. 2008, req. $n^{\circ} 36391 / 02$; CEDH Dayanan c/ Turquie, 13 oct. 2009, req. n 7377/03 ; CEDH Brusco c/ France, 14 oct. 2010, req. n 1466/07.

7. Décision $n^{\circ} 2010-14 / 22$ QPC Daniel W. et autres du 30 juillet 2010.

8. Cass. Crim. 19 octobre $2010 n^{\circ} 10-82901,10-82306$ et 10-85051.

9. http://www.syndicat-magistrature.org/Arret-de-la-CJUE-sur-la.html

10. Ordonnance 29 juin 2011, Alexandre Achughbabian c/ Préfet du Val de Marne RG $\mathrm{n}^{\circ} \mathrm{B} 11 / 02792$.

11. http://www.syndicat-magistrature.org/IMG/pdf/Achughbabian.pdf.

12. E. Fortis, «Chronique de droit pénal général », RSC 2012. 135 ; J.-H. Robert, « Divergence entre le Conseil constitutionnel et la Cour de justice de l'Union européenne », Dr. pénal 2012. Comm 34 ; A. Levade, «QPC et "directive retour" : retour en arrière jurisprudentiel ? - Décision n 2011-217 QPC du Conseil constitutionnel du 3 février 2012 », JCP G 2012.198; S. Slama, "Le Conseil constitutionnel se place en situation irrégulière au regard du droit de l'UE », ADL du 9 févr. 2012 ; D. Symon, « Directive retour et sanctions pénales du séjour irrégulier. - Le Conseil constitutionnel dans sa décision du 3 février 2012 refuse de censurer la loi française ", Europe 2012. Repère 3 ; V. Tchen, Dr. adm. 2012, Comm. 38. ; C. Saas, AJ pénal 2012, p. 410.

13. Crim., 5 juin 2012, avis n 9002, AJDA 2012. 1132 ; Gaz. Pal. 10-12 juin 2012, p. 9, note C. Pouly ; S. Slama, B. Francos, «Contrariété au droit de l'UE du placement en garde à vue d'un ressortissant d'un pays tiers sur le seul fondement du séjour irrégulier », ADL du 20 juin 2012.

14. Cass. Civ. 1 n 11-19250 ; Dalloz 2012 Actu. 1827 ; Serge Slama Recueil Dalloz 2012, p. 2001.

15. Comme le soulignait le Syndicat de la magistrature dans un communiqué daté du 6 juillet 2012 : http://www.syndicat-magistrature.org/Fin-des-gardes-a-vue-pour-les-sans.html

16. Cf. lettre ouverte du Syndicat de la magistrature au garde des Sceaux du 11 septembre 2012 et note d'analyse jointe :

http://www.syndicat-magistrature.org/IMG/pdf/lettre_ouverte_GDS_audition_libre.pdf http://www.syndicat-magistrature.org/IMG/pdf/Analyse_audition_libre_etrangers.pdf

17. Cass. Civ. 1, 14 juin 2005, 04-50.063 ; Cass. Civ. 1, 25 novembre 2009, 08-20.294.

18. Cass. Civ. 1, 21 novembre 2012, 11-30.459.

19. 23 novembre 2010, Moulin contre France, Req. $N^{\circ} 37104 / 06$.

20. L. Masera, «Les ressorts de l'affaire El Dridi », in Gisti, Un régime pénal d'exception, op. cit., p. 128. 


\section{AUTEUR}

\section{PATRICK HENRIOT}

Substitut général à la Cour d'appel de Paris et membre du Syndicat de la magistrature 